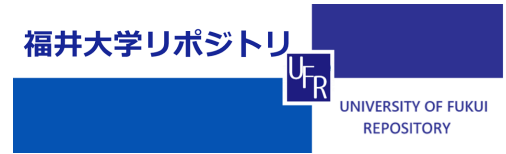

Temper at ure dependence of inf $r$ ar ed $r$ ef I ect ance spectra of I nN

\begin{tabular}{|l|l|}
\hline 著者 & $\begin{array}{l}\text { KURI HARA K, YANAGAWA T, NAKAGAWA N, FUKUI K, } \\
\text { YAMAMDTO A }\end{array}$ \\
\hline $\begin{array}{l}\text { j our nal or } \\
\text { publ i cat i on t i t l e }\end{array}$ & I NFRARED PHYSI CS \& TECHNOLOGY \\
\hline vol une & 51 \\
\hline number & 5 \\
\hline page r ange & $482-484$ \\
\hline year & 2008 05 \\
\hline URL & ht t p: //hdl . handl e. net /10098/2120 \\
\hline
\end{tabular}




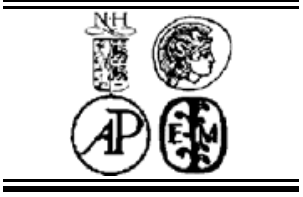

\title{
Temperature dependence of infrared reflectance spectra of InN
}

\author{
K.Kurihara ${ }^{1}$, T.Yanagawa ${ }^{1}$, N.Nakagawa ${ }^{1}$ K.Fukui $^{1 *}$, and A.Yamamoto ${ }^{2}$ \\ ${ }^{1}$ Res. Cent. Develop. Far-IR Region, Univ. Fukui, Fukui, Fukui 910-8507, Japan \\ ${ }^{2}$ Dept. Elec. Elec. Engi., Univ. Fukui, Fukui, Fukui 910-8507, Japan
}

Elsevier use only: Received date here; revised date here; accepted date here

\begin{abstract}
To investigate both the optical and electrical properties of $\mathrm{InN}$, we have measured the infrared reflectance spectra of InN thin films and performed the fitting analyses of the infrared spectra to obtain not only phonon frequencies and the damping factors but also the carrier concentration of InN. In this paper, we extend the aim of those analyses to the electron mobility and demonstrate that the temperature dependence of the electron mobility can be discussed using the infrared reflectance spectra analyses.
\end{abstract}

Keywords: InN, infrared reflectance spectra, electric mobility ;

\section{Introduction}

III-V nitride semiconductors and there mixed crystal semiconductors are used for promising high intensity light emitting diodes (LED) and laser diodes (LD). Especially, InN has a matched bandgap for the fiber communication wavelength, and it also has small effective mass and high electron mobility which are required for high speed electronic devices. However, it is difficult to grow bulk InN crystals. The high quality crystalline InN thin films have been fabricated by the MBE or MOCVD methods, but the lattice mismatch between $\mathrm{InN}$ and the substrate (normally $\mathrm{Al}_{2} \mathrm{O}_{3}$ ) requires the buffer layer. This layer is usually made of the low temperature growth $\mathrm{InN}$ or $\mathrm{GaN}$, which means that the InN sample at least consists of three layers, InN crystalline layer, buffer layer, and substrate. Then, the electric properties of the InN sample are sometime affected by this buffer layer and it is difficult to separate the electric properties of InN crystalline layer from those of the buffer layer. Then, the infrared (IR) reflectance measurements have been investigated as one of the complementary measurement methods for obtaining the carrier concentration of the InN crystalline layer [1-3]. In this method, since the dielectric constant is described by the phonons and plasmon which are characterized by each layer, the reflectance spectra are analyzed by the fitting calculations based on the appropriate layer model.

In this paper, we extend the aim of those analyses to the electron mobility and demonstrate that the

\footnotetext{
* Corresponding author. Tel.:+81-776-27-8562; fax:+81-776-27-8770; e-mail: fukui@fir.fukui-u.ac.jp.
} 
temperature dependence of the electron mobility can be discussed using the infrared reflectance spectra analyses.

\section{Experiment}

All InN samples were grown on the sapphire substrates by the MOVPE method [4]. The thicknesses of all samples are about $0.2 \sim 0.5 \mu \mathrm{m}$. The carrier concentration measurements by using the normal electric methods are carried out by the van der Pauw method $[5,6]$.

The IR reflectance spectra and their temperature dependence have been measured at the IR beamline (BL6B) of UVSOR-II (Institute for Molecular Science, Okazaki, Japan) by using both MartinPuplett type Fourier Transform Far IR (FTFIR) and Michelson type FTIR spectrometers. The wavenumber range and resolution are $50 \sim 1500 \mathrm{~cm}^{-1}$ and $1 \sim 4 \mathrm{~cm}^{-1}$, respectively, and the temperature range is $17 \sim 300 \mathrm{~K}$.

The IR spectra have been analyzed by the numerical simulations. The fitting calculations are carried out by using Eq. (1) to (3) [7]. The complex dielectric constant in the IR region is usually described as the $\mathrm{LO}$ and TO phonons frequencies $\left(\omega_{\mathrm{L}}\right.$, $\left.\omega_{\mathrm{I}}\right)$ and their damping factor $\Gamma$. However, the contribution of the electric carriers must be considered when the plasma frequency is located in the IR region. In such a case, the dielectric constant including the coupling between plasmon and phonons are represented by Eq. (1),

$$
\varepsilon(\omega)=\varepsilon_{\infty}\left[1+\frac{\omega_{L}{ }^{2}-\omega_{T}{ }^{2}}{\omega_{T}{ }^{2}-\omega^{2}-i \omega \Gamma}-\frac{\omega_{p}{ }^{2}}{\omega(\omega+i \gamma)}\right]
$$

where $\omega_{\mathrm{P}}$ and $\gamma$ are plasma frequency and damping factor of the plasmon, respectively. The $\omega_{\mathrm{p}}$ and $\gamma$ are also described by Eq. (2) and (3),

$$
\begin{gathered}
\omega_{P}{ }^{2}=\frac{4 \pi n e^{2}}{m^{*} \varepsilon_{\infty}} \\
\gamma=\frac{e}{m^{*} \mu}
\end{gathered}
$$

where $m^{*}, n$ and $\mu$ are effective mass, carrier concentration, and mobility of the carriers, respectively. The dielectric constant of the sapphire substrate is simply represented by the contributions of the phonons. Then, by using the transfer matrix method, the theoretical total reflectance including the multiple reflection based on any layer model can be described, and the fitting analyses to the observed reflectance spectra are available using the physical values $\omega_{\mathrm{L}}, \omega_{\mathrm{\Gamma}}, \omega_{\mathrm{P}}, \gamma, \Gamma, \varepsilon_{\infty}$ as the fitting parameters.

\section{Result and discussion}

Fig. 1 shows the typical reflectance spectrum of the InN thin film at $300 \mathrm{~K}$ and the fitting curve. The carrier concentration measured by Van der Pauw method is $9.8 \times 10^{18} \mathrm{~cm}^{-3}$. The fitted curve shown in Fig. 1 is based on the two layers model which means no consideration of the buffer layer in the optical point of view and in agreement with the observed spectrum. The fitting parameters are $\varepsilon_{\infty}=8.3, \omega_{\mathrm{L}}=$ $581 \mathrm{~cm}^{-1}, \omega_{\mathrm{T}}=478 \mathrm{~cm}^{-1}, \omega_{\mathrm{P}}=836 \mathrm{~cm}^{-1}, \gamma=265, \Gamma=$ 2 and $h$ (thickness of crystalline InN layer) $=200 \mathrm{~nm}$. Since the effective mass is still unknown for $\mathrm{InN}$, we suppose it is $0.1 m_{0} \sim 0.15 m_{0}$ according to the theoretical prediction [8], where $m_{0}$ is the static mass of the electron. The carrier concentration derived from IR reflectance spectrum is estimated to $6.5 \sim 9.7$ $\times 10^{18} \mathrm{~cm}^{-3}$, which is a little smaller than Van der Pauw result. However, from this result, it is concluded that the electric properties of this InN sample are not affected by those of the buffer layer.

From Eq. (3), the electron mobility can be derived from the IR reflectance measurement by using the damping factor $\gamma$ of the plasmon which is one of the

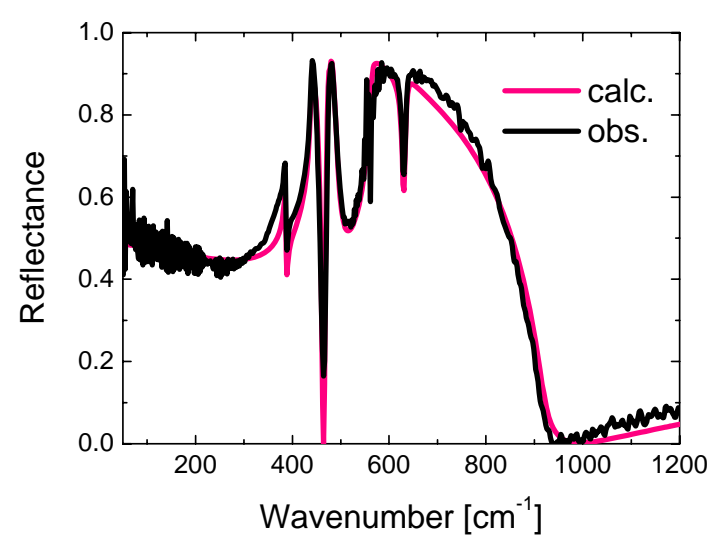

Fig. 1: Typical IR reflectance spectrum of $\mathrm{InN}$ thin film $\left(9.8 \times 10^{18} \mathrm{~cm}^{-3}\right)$ and fitting curve. 


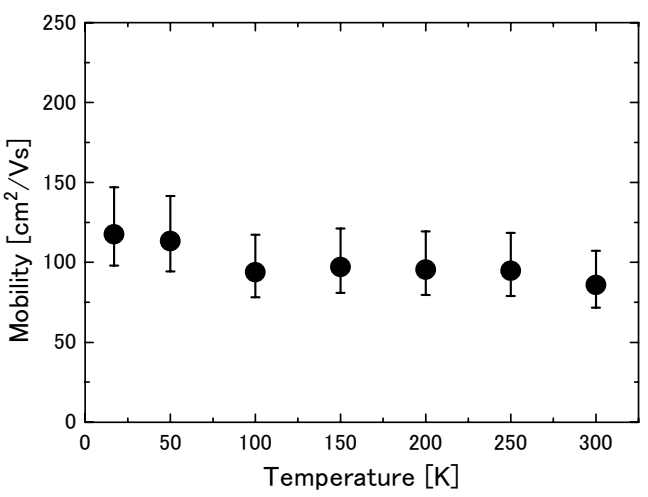

Fig. 2: The electron mobility of the InN crystalline layer derived from the infrared reflectance measurements as a function of the temperature.

fitting parameters in Eq. 1 and the effective mass $m^{*}$. Fig. 2 shows the electron mobility of the $\mathrm{InN}$ crystalline layer derived from the IR reflectance measurements as a function of the temperature. The electric mobility of $\mathrm{InN}$ is almost constant in the whole temperature range, and is in agreement with the results of the Hall effect measurement. The inverse of the total electric mobility is described as the summation of the inverse mobilities of the scattering processes, and it is known that both the polar phonon scattering and the deformation potential scattering have $\mathrm{T}^{-2 / 3}$ temperature dependences, and the ionized impurity scattering has $\mathrm{T}^{3 / 2}$ dependence $[9,10]$. However, it is difficult to explain such a constant behavior in the wide temperature range. It seems that the additional contributions, for example, the space charge scattering should be considered. This analysis is still under investigation.

\section{Conclusion}

We have measured the infrared reflectance spectra of the InN thin films at the temperature range of 11 to $300 \mathrm{~K}$. Since the damping factor of the plasmon which is one of the fitting parameters of the infrared reflectance spectrum analysis is reciprocal proportion to the electron mobility, we demonstrate that the electron mobilities of the $\mathrm{InN}$ crystalline layers and their temperature dependence can be discussed using the infrared reflectance spectra analyses. The electron mobilities of the InN crystalline layers are almost independent on the temperature in the range of 11 to
$300 \mathrm{~K}$, and it is difficult to explain this flatness by using both phonon and impurity scattering processes.

\section{Acknowledgements}

This work was partly supported by the Join Studies Program (2006-2007) of UVSOR facility, Institute for Molecular Science.

\section{References}

[1] Y. Ishitani, K. Xu, W. Terashima, N. Hashimoto, M. Yoshitani, T. Hara, and A. Yoshikawa, phys. stat. sol. (c) 0, 2838 (2003).

[2] J. S. Thakur, G. W. Auner, D. B. Haddad, R. Naik, and V. M. Naik, J. Appl. Phys. 75, 4795 (2004).

[3] K. Fukui, Y. Kugumiya, N. Nakagawa, and A. Yamamoto, phys. stat. sol. (c) 3, 1879 (2006).

[4] A. Yamamoto, K. Sugita, H. Takatsuka, A. Hashimoto, and V. Yu. Davydov, J. Cryst. Growth 261, 275 (2004).

[5] L. J. van der Pauw, Philips Res. Repts. 13, 1 (1958).

[6] L. J. van der Pauw, Philips Tech. Rev. 20, 220 (1958).

[7] M. Kuball, Surf. Interface. Anal. 31, 98 (2001).

[8] J.Wu and W. Walukiewicz., Superlattice. Microst. 34, 63 (2003).

[9] J. Bardeen, W. Shockley, Phys. Rev. 80, 72 (1950).

[10] W.A. Harrison, Phys. Rev. 100, 255 (1956). 